\title{
BUSINESS INTELLIGENCE: UM ESTUDO SOBRE O NÍVEL DE MATURIDADE EM EMPRESAS DE CONFECÇÕES DE LINGERIE
}

\author{
Paulo César dos SANTOS ${ }^{1}$ \\ Marly de Souza PRADO ${ }^{2}$
}

IFSULDEMINAS - Câmpus Muzambinho. paulo.santos@ifsuldeminas.edu.br
IFSULDEMINAS - Câmpus Muzambinho. marlylica@ gmail.com

Recebido em: 22/12/2013 - Aprovado em: 15/09/2014 -Disponibilizado em: 15/12/2014

Resumo:Business Intelligence (BI) é um processo para geração de sistema de apoio a decisão dentro das organizações, tem a finalidade de produzir relatórios dinâmicos que auxiliam as tomadas de decisões estratégicas. Este trabalho teve por objetivo avaliar o uso de sistemas de BI nas indústrias de confecções de Lingerie em Juruaia - MG, através da verificação do nível de maturidade. Os resultados encontrados apontaram que a metade das empresas entrevistadas possui um sistema com módulo de BI e a outra metade apenas possui sistemas transacionais. Foi observado também que a maioria das empresas foi classificada no nível 2 de maturidade.

Palavras-chave: Sistema de informação. Inteligência nos Negócios. Armazém de Dados. BI. Nível de Maturidade.

\begin{abstract}
Business Intelligence (BI) is a process for the generation of the decision support system within organizations, aims for producing dynamic reports aiding strategic decision making. This study aims to evaluate the use of BI systems in industries confections Juruaia - MG by checking the maturity level. The results showed that half of the respondents have a system with BI module and the other half has just transactional systems. In medium industries were classified as level 2 maturity.
\end{abstract}

Keywords: Information System. Business Intelligence. Data wharehouse. BI. Maturity Level.

\section{Introdução}

O processo de Business Intelligence (BI), que em tradução livre significa inteligência nos negócios, está relacionado com o sistema de informação estratégica (SIE) da organização. É utilizado para auxiliar a tomada de decisões, desde a alta gerência até as gerências operacionais. Permite analisar os dados de forma mais assertiva, criando informação baseada em dados concretos por meio de métricas em relatórios gerados por sistemas automáticos (PRIMAK, 2008).

Os relatórios gerados pelo processo de BI estão baseados no conceito de Data
Warehouse (DW), banco de dados multidimensional. De acordo com Elmasri e Navathe (2011), são sistemas de grande importância para as organizações exigindo anos na conceitualização e implantação do projeto, para atingirem o objetivo de extração de informações.

Atualmente a informação possui um valor significativo tanto para pessoas quanto para instituições, pois, através das mesmas podem ser adquiridas vantagens competitivas. Informações valiosas são adquiridas por um processo de transformação dos dados, que serão selecionados e utilizados para conhecer 
ainda, verificar as consequências das decisões tomadas (REZENDE; ABREU, 2011).

A análise de dados é uma atividade em expansão, necessária para a garimpagem de padrões dentro dos armazéns de dados das instituições, na década de 2010 o volume de dados está na escala do petabytes. A elevação do volume de armazenamento de dados teve influência com a internet e levou ao surgimento de novos conceitos como o Business Intelligence 2 (BI2). Esse procura estreitar a latência de tempo de acesso à informação e abrangem também novas aplicações de tecnologias, como os sistemas para dispositivos móveis e métodos ágeis (BARBIERI, 2011).

Além da aplicação de novas tecnologias, Barbieri (2011) afirma que o BI já se encontra em uso em diversos tipos de organizações no Brasil, com aplicações interessantes em empresas varejistas, operadoras de saúde, entre outras.

O crescimento no uso do processo de BI nas organizações, conciliado com $\mathrm{o}$ compromisso de desenvolvimento regional do IFSULDEMINAS (2012), motiva explorar a utilização dessa tecnologia na região de Muzambinho. O intuito é conhecer melhor o desenvolvimento tecnológico regional e criar um referencial para futuros estudos, uma vez que será possível obter um parâmetro sobre a utilização desses conceitos na região.

Este artigo tem como objetivo apresentar os resultados da avaliação dos sistemas de informação e a análise do nível de maturidade no uso de BI nas indústrias de confecções de Lingerie no município de Juruaia - MG.

Essas indústrias surgiram com a organização de um polo produtor, que foi estruturado desde a década de 1990. Atualmente esse município é responsável pela produção de $15 \%$ do total de Lingeries fabricadas no Brasil, emprega aproximadamente 3.500 pessoas em mais de 150 confecções, segundo a Associação Comercial e Industrial de Juruaia (ACIJU, 2013).

$\mathrm{O}$ artigo descreve a metodologia para a pesquisa, os resultados obtidos, a conclusão e as referências bibliográficas utilizadas.

\section{Metodologia}

Inicialmente foram realizados estudos sobre trabalhos correlatos a essa proposta. $\mathrm{Na}$ esfera internacional pode-se citar Côrte-Real (2010) que propôs uma metodologia de avaliação de maturidade de BI em uma organização, com intuito de avaliar as iniciativas nas organizações.

Pelissari et al. (2012) apresentaram um artigo sobre o mercado de confecções na cidade de Vila Velha - ES. Nesse, foi abordado o conceito de Inteligência Competitiva que também é uma área de apoio à tomada de decisão. 
Existem estudos na área, como o de Souza (2011) sobre a aplicação de BI em um departamento de controladoria de uma empresa de agronegócio na região do Sul de Minas Gerais.

O método de pesquisa utilizado foi proposto por Wazlawick (2011) no livro Metodologia de Pesquisa para Ciência da Computação.

Foi realizada uma pesquisa quantitativa de natureza descritiva e caráter exploratório, para identificar as organizações que utilizam o processo de BI em seus sistemas de informação.

Para a pesquisa quantitativa, foram selecionadas 12 empresas que empregam entre 10 a 200 colaboradores. Foram entrevistados proprietários e gerentes. Os nomes das mesmas não serão citados.

O nível de maturidade foi determinado de acordo com o proposto por Getz (2013), a seguir apresenta-se a tabela de níveis de 0 a 5, onde 0 é um baixo nível de uso da tecnologia e 5 é um alto nível de uso da tecnologia.

Tabela 1 - Níveis de maturidade de um sistema de BI

\begin{tabular}{l|l}
\hline Níveis & Descrição \\
\hline Nível 0 - BI Limitado & $\begin{array}{l}\text { Utilização de planilhas } \\
\text { eletrônicas para controle } \\
\text { interno. }\end{array}$ \\
\hline $\begin{array}{l}\text { Nível 1 - Relatórios } \\
\text { operacionais }\end{array}$ & $\begin{array}{l}\text { Utilização de relatórios } \\
\text { operacionais padronizado } \\
\text { pelo sistema, vindos de } \\
\text { um banco de dados } \\
\text { transacional. }\end{array}$ \\
\hline Nível 2 - Análises e \\
consultas & $\begin{array}{l}\text { Utilização de relatórios } \\
\text { customizáveis pelo } \\
\text { usuário, vindos de um } \\
\text { banco de dados } \\
\text { transacional. }\end{array}$ \\
\hline Nível 3 - Painéis & Utilização de gestão de \\
\hline
\end{tabular}

\begin{tabular}{l|l}
\hline interativos & $\begin{array}{l}\text { painéis, vindos de um } \\
\text { Data Wharehouse (DW). }\end{array}$ \\
\hline Nível 4 - OLAP & $\begin{array}{l}\text { Utilização de relatórios } \\
\text { por Online Analitical } \\
\text { Processing (OLAP), } \\
\text { vindos de um DW. }\end{array}$ \\
\hline Nível 5 - Data Mining & $\begin{array}{l}\text { Utilização de painéis de } \\
\text { previsão e mineração de } \\
\text { dados, vindos de um DW. }\end{array}$ \\
\hline
\end{tabular}

Fonte: Elaborada pelos autores.

$\mathrm{O}$ instrumento utilizado para a coleta de dados foi um questionário estruturado com 11 perguntas para obter resposta objetivas. As questões foram desenvolvidas com o objetivo de buscar evidências de um projeto de BI, a utilização de suas ferramentas ou o uso de seus conceitos e ainda determinar o nível de maturidade, de acordo com a Tabela 1 .

O questionário foi apresentado aos entrevistados em visita às empresas, durante o mês de abril de 2013 e disponibilizado eletronicamente para ser respondido. Houve um retorno de respostas de $100 \%$ dos entrevistados. Depois de aplicado, foi gerado um conjunto de informações, para avaliações e análises.

\section{Resultados}

Para apuração dos resultados foram analisados e apresentados os dados de acordo com o método estatístico descritivo, com o objetivo de descobrir qual o nível geral dos sistemas de informação e identificar a necessidade de sistemas mais avançados para a região

As informações serão demonstradas através de gráficos e acompanhadas de uma análise textual. 
O Gráfico 1 mostra o porte das empresas pesquisadas, de acordo com a quantidade de funcionários. Observa-se que $75 \%$ das confecções possuem até 50 funcionários, $17 \%$ possuem entre 50 a 100 funcionários e $8 \%$ possuem entre 100 a 200 funcionários.

Gráfico 1 - Quantidade de funcionários.

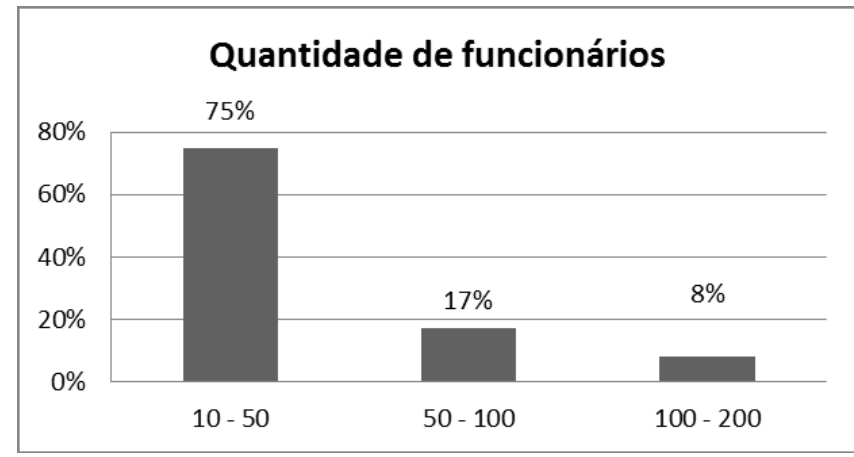

Fonte: Elaborado pelos autores

O Gráfico 2 evidencia que $64 \%$ das confecções pretendiam investir em sistemas de informação e $36 \%$ não pretendiam investir.

Gráfico 2 - Investimento em sistemas de informação.

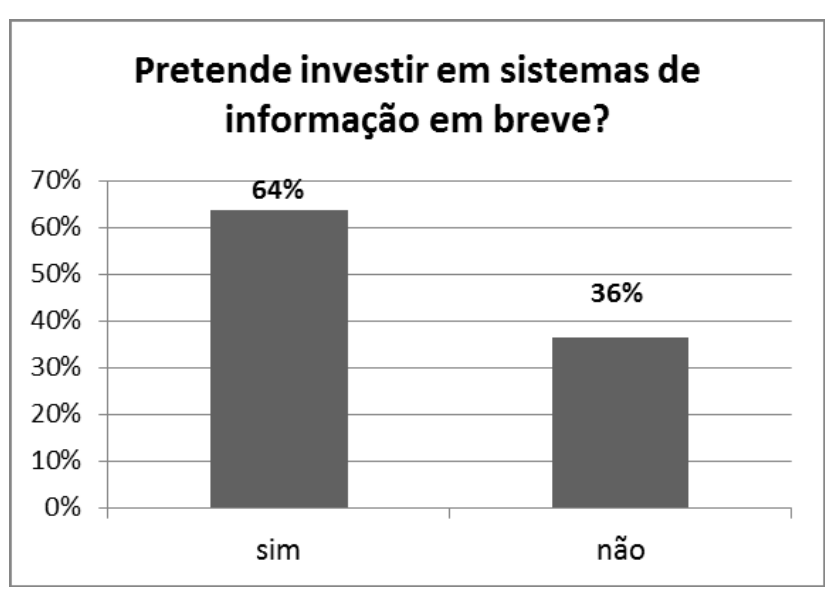

Fonte: Elaborado pelos autores
O Gráfico 3 mostra que $75 \%$ dos administradores conheciam ou já ouviram falar sobre inteligência nos negócios e $25 \%$ desconheciam.

Gráfico 3 - Inteligência nos negócios

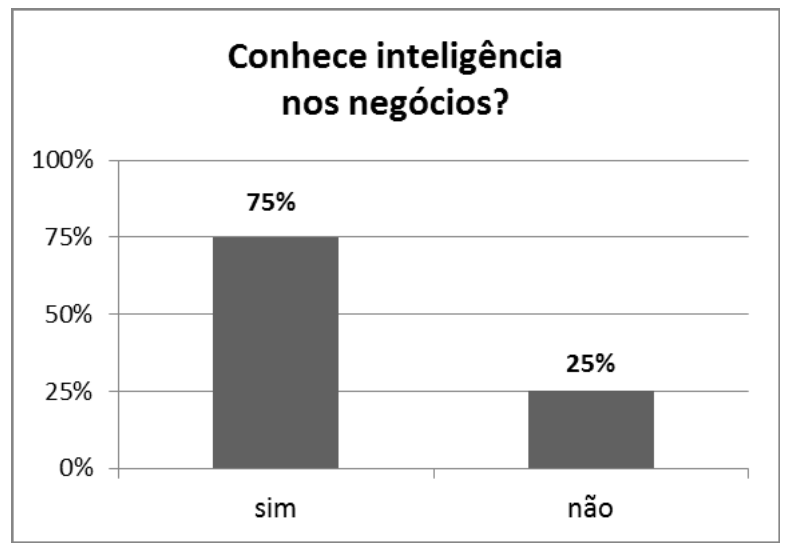

Fonte: Elaborado pelos autores

O Gráfico 4 mostra que os administradores conheciam os relatórios prontos das técnicas de OLAP (relatórios temporais 83\%), mineração (descoberta de dados 67\%) e identificação de tendências $58 \%$ ), mas, apenas $25 \%$ conheciam os painéis interativos.

Gráfico 4 - Tipos de relatórios

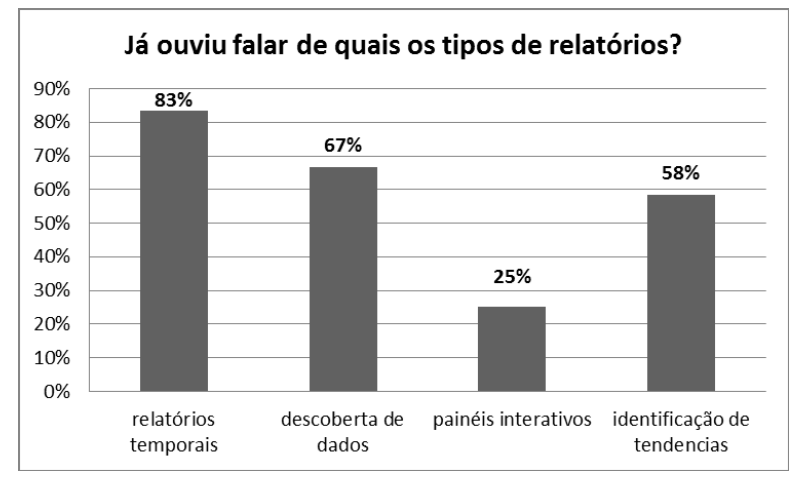

Fonte: Elaborado pelos autores 
O Gráfico 5 apresenta 3 sistemas utilizados pelas empresas, deste ponto em diante, serão citados apenas pelos nomes representativos: Sistema A para o Millennium, Sistema B para o Cacique e Sistema C para o Empresário. O Sistema A é utilizado por $50 \%$, o Sistema B por $33 \%$ e o Sistema C por $17 \%$ das empresas.

Após a obtenção destes resultados foram analisadas as características técnicas de cada um dos sistemas. Foi observado que todos são Enterprise Resource Planning (ERP) especializados para a indústria de confecções e com o mesmo tipo de banco de dados. O sistema A possui um módulo de BI interno e os sistemas B e C não possuem.

Gráfico 5 - Sistemas das empresas.

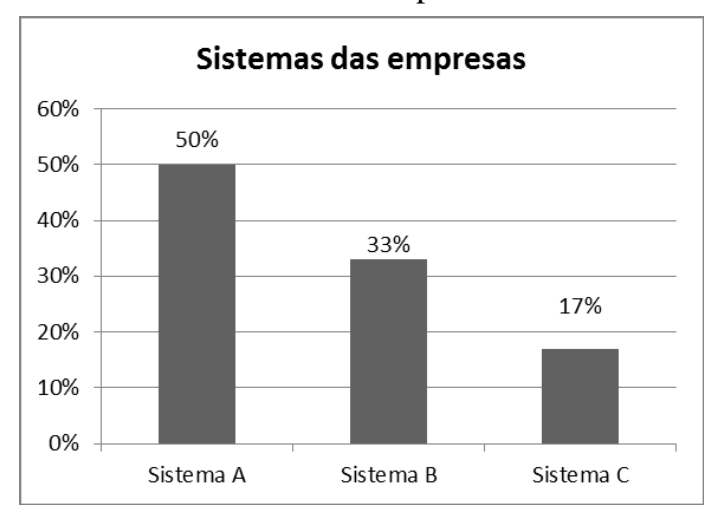

Fonte: Elaborado pelos autores

O Gráfico 6 mostra que 92\% dos entrevistados possuíam um controle de entrada de dados no sistema e $8 \%$ não possuem.
Gráfico 6 - Governança na qualidade de dados.

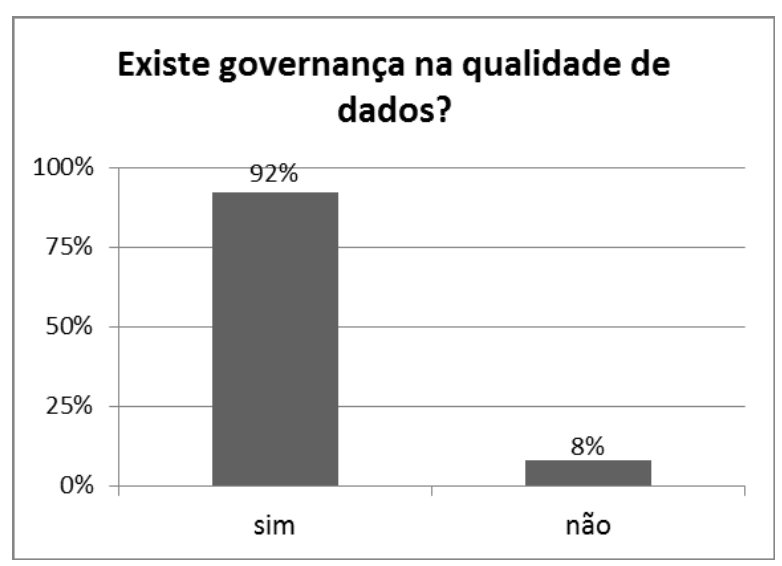

Fonte: Elaborado pelos autores

O Gráfico 7 apresenta o levantamento do departamento com maior necessidade de utilização de relatórios. O departamento Financeiro foi apontado com 83\%, seguido pelos departamentos de Produção e Vendas com $75 \%$, Clientes com $33 \%$ e Marketing $8 \%$.

Gráfico 7 - Departamentos com maior necessidade de relatórios.

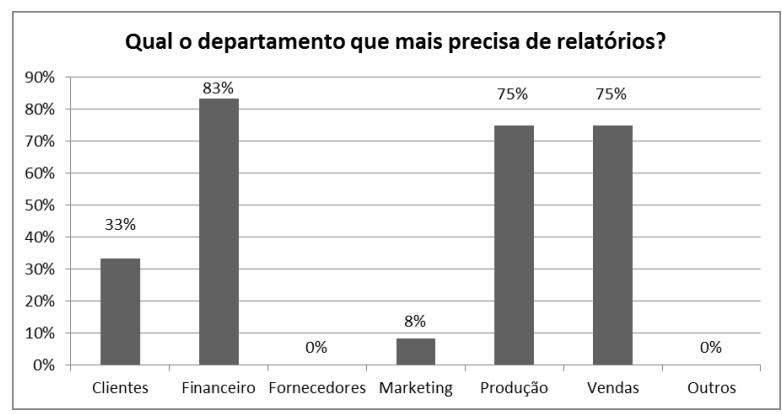

Fonte: Elaborado pelos autores

O Gráfico 8 demonstra como é tratado o serviço de TI nas empresas, $42 \%$ existe um profissional técnico, em $50 \%$ apontaram que existe apenas um serviço terceirizado e em $8 \%$ existe um gestor de TI. 
Gráfico 8 - Existência de um departamento de TI.

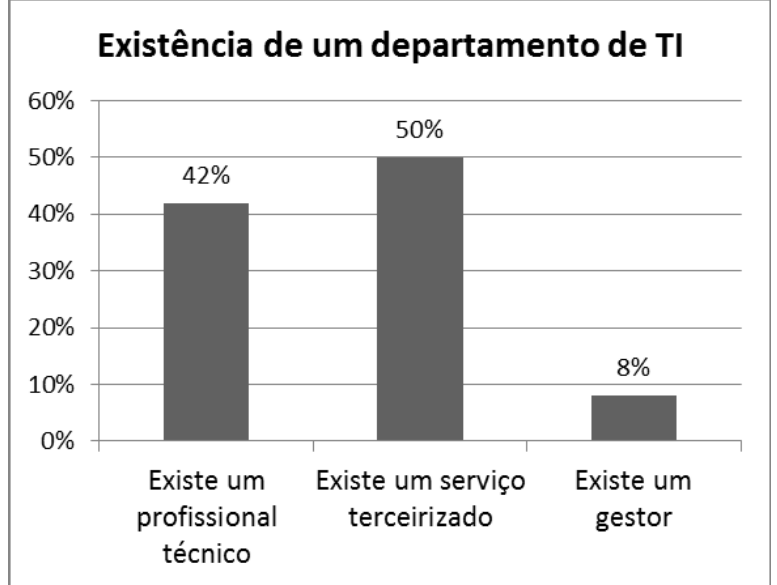

Fonte: Elaborado pelos autores

O Gráfico 9 apresenta a quantidade de empresas em cada nível de maturidade de BI, observa-se que $100 \%$ das empresas possuem algum indício de BI. No nível 0 encontram-se 17\% das empresas; no nível 1 25\%; no nível 2 encontra-se a maior parte das empresas com 33\%; no nível 3 17\%; e no nível 4 foram 8\%.

Gráfico 9 - Nível de maturidade.

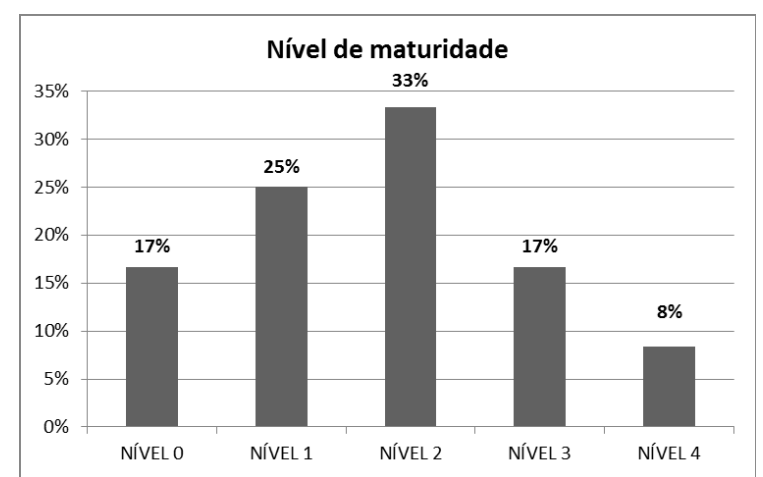

Fonte: Elaborado pelos autores

As empresas de nível 2 estão no grupo predominante com $42 \%$, essas possuem um serviço terceirizado de TI. No grupo das empresas de nível 4, maior índice alcançado, são as mesmas empresas que possuem um gestor de TI interno.

\section{Conclusão}

Após a execução deste trabalho concluiu-se que as empresas pesquisadas possuem sistemas que podem ser utilizados para aplicação dos conceitos de BI, esses podem apoiar as estratégias de negócios nas empresas pesquisadas. A maior parcela das empresas (33\%), encontra-se no nível 2 de maturidade, de acordo com os níveis propostos por Getz (2013).

Embora existam os sistemas que atendem aos requisitos necessários para $\mathrm{BI}$, há falta de profissionais técnicos internos qualificados para a boa utilização dos mesmos. Faltam também conhecimentos técnicos relacionados aos processos de BI. A pesquisa apontou que as empresas que possuem gestores de TI obtiveram melhores resultados no nível de maturidade de BI.

Este foi um dos primeiros estudos do IFSULDEMINAS - Câmpus Muzambinho sobre sistemas de informação na região pesquisada e gerou um artefato que poderá ser utilizado inclusive para analisar sistemas de informação em empresas de outros segmentos industriais.

Após essas conclusões sugere-se a continuidade do estudo por meio de trabalhos futuros, com intuito de acompanhar a implantação de um projeto de BI em algumas das empresas que ainda não utilizam esta tecnologia. 


\section{Referências bibliográficas}

ACIJU - Associação Comercial e Industrial de Juruaia. Disponível em:

<http://www.aciju.com.br>. Acesso em: 07 mar. 2013.

\section{BARBIERI, Carlos. BI2 - Business}

Intelligence: modelagem e qualidade. 1. ed. Rio de Janeiro: Elsevier, 2011.

CÔRTE-REAL, Nadine. Avaliação da maturidade da businessintelligence nas organizações. Universidade Nova de Lisboa, 2010.

ELMASRI, Ramez; NAVATHE, Shamkant B.. Sistemas de banco de dados.6. ed. São Paulo: Person Addison Wesley, 2011. 788 p.

GETZ, Adam. Business Intelligence (BI)

Maturity Model.Disponível em: <http://biinsider.com/portfolio/bi-maturity-model/>. Acesso em: 07 mar. 2013.

IFSULDEMINAS. O instituto. Disponível

em:

<http://www.ifsuldeminas.edu.br/index.php/pt /o-instituto>. Acesso em 10 dez. 2012.

PELISSARI, Anderson Soncini et al. Diagnóstico do uso da inteligência competitiva empreendedora em pequenas empresas da indústria de confecções da cidade de Vila Velha - ES. Rev. Adm. Ufsm, Santa Maria, n., p.182-203, 01 ago. 2012. Quadrimestral.

PRIMAK, Fábio Vinicius da Silva. Decisões com B.I. (Business Intelligence). 1. ed. Rio de Janeiro: Editora Ciência Moderna Ltda., 2008.

REZENDE, Denis Alcides; ABREU, Aline França de. Tecnologia da Informação aplicada a sistema de informação empresarial: o papel estratégico da informação e dos sistemas de informações nas empresas. 8. ed. São Paulo: Atlas, 2011.

SOUZA, Patrícia Conceição de. Um estudo formal da tecnologia de business intelligence BI. Trabalho de Conclusão de
Curso (Curso Ciência da Computação) Unifeg, Guaxupé, 2011.

Wazlawick,Raul Sidnei. Metodologia de Pesquisa para Ciência da Computação. 6 . ed. Rio de Janeiro: Elsevier, 2011. 